\title{
Adult stem cells: potential implications for perioperative medicine
}

\author{
Manoj M. Lalu, MD, PhD • Carly C. Barron, MSc • \\ Duncan J. Stewart, MD • Lauralyn L. McIntyre, MD
}

Received: 4 December 2013/ Accepted: 23 January 2014/Published online: 8 February 2014

(C) Canadian Anesthesiologists' Society 2014

In May 2012, Health Canada approved the use of mesenchymal stromal cells for the treatment of severe pediatric graft-versus-host disease, ${ }^{\mathrm{A}}$ making Canada the first country in the world to approve a stem cell therapy. This important decision heralded the arrival of an important new modality for the treatment of inflammatory disease.

\section{What are mesenchymal stromal cells?}

Since their initial description by Friedenstein et al. over 40 years ago, ${ }^{1}$ mesenchymal stromal cells (MSCs) have been referred to by a number of terms, including mesenchymal stem cells and "adult stem cells". Friedenstein's pioneering studies of MSCs described their adherence to untreated culture dishes, their spindle-shaped morphology, as well as their ability to differentiate into chondrocytes, adipocytes, and osteocytes under certain conditions. Although these basic criteria continue to define MSCs, ${ }^{2}$ our understanding of these cells has greatly evolved. Of importance, MSCs, unlike embryonic stem cells, are not a

M. M. Lalu, MD, PhD (ه) · C. C. Barron, MSc

Department of Anesthesiology, The Ottawa Hospital Research Institute, University of Ottawa, Civic Campus, Room B307,

1053 Carling Avenue, Mail Stop 249, Ottawa, ON K1Y 4E9, Canada

e-mail: manojlalu@gmail.com

\section{J. Stewart, MD}

Regenerative Medicine Program, The Ottawa Hospital Research Institute, University of Ottawa, Ottawa, ON, Canada

L. L. McIntyre, MD

Division of Critical Care, Department of Medicine, University of

Ottawa, Ottawa, ON, Canada single cell type capable of differentiating into multiple cell types in vivo; instead, MSCs are a heterogeneous population of cells capable of a broad number of biological activities. Thus, it has been suggested that the term, "stromal cell", be applied to MSCs as they do not display prototypical stem cell characteristics. ${ }^{3}$

Initially, MSCs were isolated from adult bone marrow (where they are believed to support hematopoiesis) as well as adipose tissue and Wharton's jelly from umbilical cords. These sources remain the best characterized; however, MSCs are now regarded to be ubiquitous cells found in virtually every tissue studied. ${ }^{4}$ Controversy exists as to the physiological role of these cells under native in vivo conditions; however, it has been suggested that MSCs may be perivascular cells ("pericytes") that support vascular homeostasis and tissue repair. In preclinical studies, perivascular MSCs appear to be mobilized into the circulation under inflammatory conditions, such as traumatic lung injury ${ }^{5}$ or hypoxia. $^{6}$ A similar mobilization of MSCs into the circulation has also been observed clinically in elderly patients following traumatic hip fracture. ${ }^{7}$

\section{MSCs as an "off-the-shelf" therapy}

Mesenchymal stromal cells can be cultured and significantly proliferate in vitro irrespective of the tissue source. When expanded to sufficient numbers, allogeneic MSCs may be processed and administered to a patient without cross-matching. Mismatched allogeneic cells have been used clinically, ${ }^{8}$ and even xenogeneic cells have been

\footnotetext{
A Health Canada. Summary Basis of Decision (SBD) for PrPROCHYMAL ${ }^{\circledR} ; 11-29-2013$.
} 
used successfully in immunocompetent preclinical models. ${ }^{9}$ This remarkable ability of MSCs to evade detection by the recipient's immune system has been attributed to the lack of major histocompatibility complex class II and co-stimulatory molecules necessary for host CD4+ T cell recognition. ${ }^{10}$ The immunoprivileged status of MSCs is clinically important as it is one factor that allows for an "off-the-shelf" product that could be administered in acute circumstances.

\section{Potential perioperative applications of MSCs}

The effects of exogenously administered MSCs in animal studies appear to be largely anti-inflammatory and organ protective; thus, MSCs could be potentially beneficial against the surgical stress and inflammation seen in the perioperative period. For example, in a preclinical model of ventilator-induced lung injury, MSCs administered intratracheally or intravenously reduced pro-inflammatory cytokine production and improved arterial oxygenation and lung compliance. ${ }^{11}$ Similarly, MSCs delivered in several large animal models of myocardial infarction have increased ejection fraction and decreased scar size. ${ }^{12}$ Mesenchymal stromal cells have also proven useful in preclinical acute kidney injury where they displayed potent anti-apoptotic and cytoprotective effects. ${ }^{13}$ Interestingly, in preclinical sepsis models, MSCs not only improved survival and organ function but also showed potent antibacterial effects. ${ }^{14}$ Given these organ protective effects as well as the ability to clear pathogens, it is clear that MSCs could have potential benefits for many patients in the perioperative period. Preoperatively, many conditions could potentially be optimized by MSCs as well (Table). To date, however, the perioperative population has been the subject of only a few MSC clinical studies; this presents a significant opportunity for future MSC clinical research.

Table Example conditions in which MSC administration may have therapeutic potential

\begin{tabular}{ll}
\hline Preclinical studies & Clinical studies \\
\hline $\begin{array}{l}\text { Ventilator-induced lung } \\
\text { injury }\end{array}$ & $\begin{array}{l}\text { Acute myocardial infarction and heart } \\
\text { failure }\end{array}$ \\
$\begin{array}{l}\text { Pulmonary hypertension } \\
\text { Infectious acute lung } \\
\text { injury }\end{array}$ & $\begin{array}{l}\text { Acute kidney injury } \\
\text { Graft-versus-host disease }\end{array}$ \\
$\begin{array}{l}\text { Bronchopulmonary } \\
\text { dysplasia }\end{array}$ & Stroke \\
$\begin{array}{l}\text { Sepsis } \\
\text { Trauma }\end{array}$ & Multiple sclerosis \\
Burn injury & Crohn's disease \\
\hline
\end{tabular}

\section{Therapeutic pathways of MSCs}

The exact mechanisms by which MSCs exert their beneficial effects remain somewhat elusive and controversial. Nevertheless, it is evident that the effects of MSCs do not rely on cell engraftment as total cell retention is extremely low even when MSCs are delivered directly into tissue (e.g., $<6 \%$ retention ten days after transendocardial injection). ${ }^{15}$ Instead, it is thought that MSCs migrate by chemotaxis to areas of inflammation ${ }^{16}$ and have pleiotropic effects through paracrine mechanisms (Figure). This was clearly shown in a preclinical model of sepsis in which MSCs were administered by intravenous injection and only very low levels of cell persistence could be established. ${ }^{17}$ Nonetheless, in this study, mRNA microarray analysis of spleen, lung, heart, and liver revealed that 4,751 genes were significantly changed in MSC-treated $v s$ placebo-treated animals. Network analysis of these transcriptional responses identified common pathways that improved mitochondrial function, dampened the pro-inflammatory response, and maintained endothelial integrity. Thus, unlike typical drug-based therapeutics which act largely via "lock-and-key" mechanisms, cellular therapy with MSCs acts via a myriad of pathways to decrease inflammation and maintain homeostasis.

The novel mechanisms by which MSCs exert their therapeutic effects have become a focus of recent research. One interesting example was found in a mouse model of acute lung injury in which human MSCs were administered intratracheally. These xenogeneic MSCs formed gap junction channels with host murine cells and transferred mitochondria through the formation of microvesicles. ${ }^{18}$ This was observed by microscopy of these transfers as well as by the presence of persistent human mitochondrial DNA in the recipient mouse lungs. Functionally, mitochondrial transfer resulted in increased adenosine triphosphate (ATP) formation and improved survival of these animals.

Aside from mitochondria, other factors released by MSCs hold potential interest. Factor concentrates can be produced from factors released by MSCs by collecting culture media in which the cells are grown. In preclinical models of acute lung injury, it has been shown that these MSC culture media concentrates may be as effective as MSCs themselves in reducing inflammation. ${ }^{11,19}$ Future research will be needed for further characterization of the therapeutic factors released by MSCs.

\section{Safety of MSCs}

Given the potentially beneficial effects of MSCs, it is not surprising that these cells have been tested in a number of 
Figure Potential therapeutic effects of mesenchymal stromal cells

Figure Effets thérapeutiques potentiels des cellules stromales mésenchymateuses

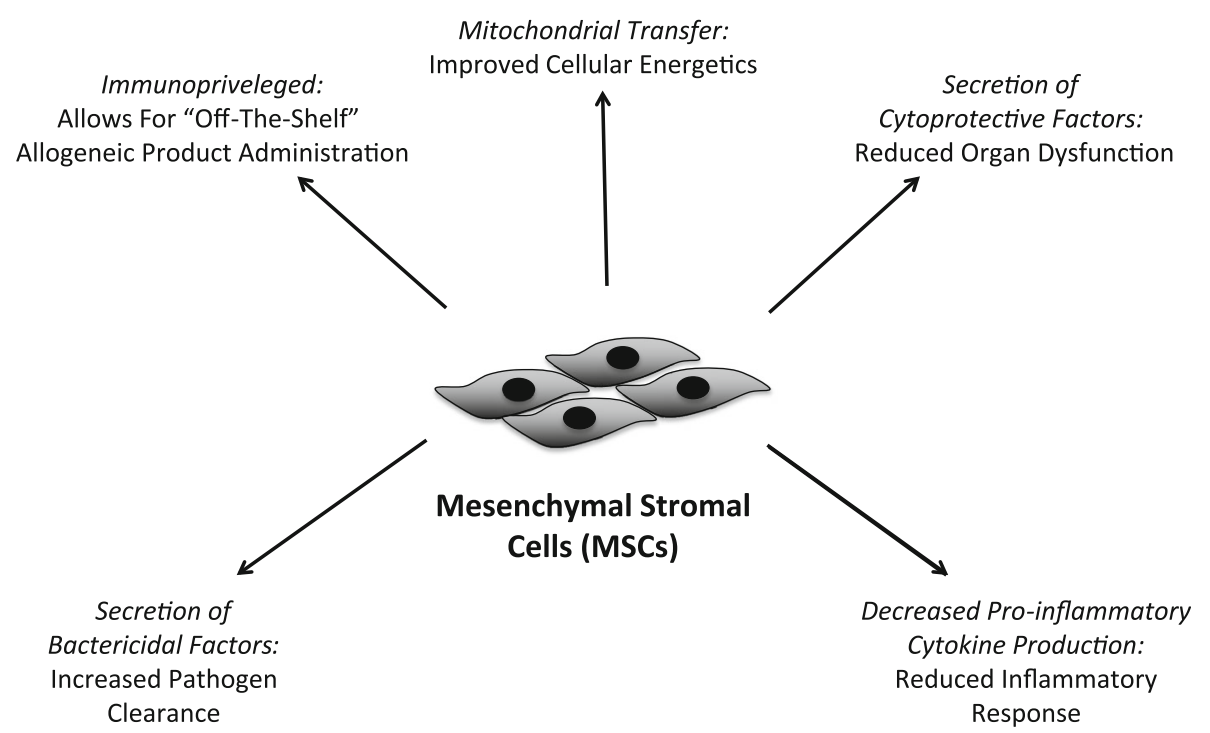

clinical conditions (Table). Mesenchymal stromal cells have been tested in clinical trials involving human subjects in various conditions, including stroke, myocardial infarction, heart failure, acute kidney injury, and steroidresistant graft-versus-host disease amongst other conditions. As with any investigational therapy, a focus on safety should remain paramount. In order to address these concerns, we recently completed a systematic review and meta-analysis on the safety of MSCs in clinical trials. ${ }^{20}$ Based on our analysis of published trials, we found no association between systemic MSC administration and adverse outcomes such as organ dysfunction and acute infusion reactions. It is noteworthy that, despite the immunosuppressive effects of MSCs, subjects did not have higher rates of infections. This is in keeping with the beneficial effects of MSCs on bacterial clearance seen in preclinical sepsis models. As well, even though MSCs are immunoprivileged and may engraft, there were no increases in tumour formation or malignancy. This review was limited, however, by the small overall number of trials and total number of patients studied, as well as the relatively short follow-up periods, and the wide range of MSC dosing schemes. Additional well-conducted and rigorous clinical studies will be needed for further clarification of the safety profile of MSCs. In future studies, there will also be the need for careful examination of the MSC dose-response relationship in various conditions.

\section{Potential implications of anesthetics on MSCs}

An important consideration for anesthesiologists in the therapeutic usage of MSCs is the potential effect of local and general anesthetics on these cells. This will be important for future patients who receive MSCs preoperatively or intraoperatively.

Perioperative use of local anesthetics may be a concern as these drugs are cytotoxic in a number of cell types, and it appears that MSCs may be susceptible as well. In a study on murine MSCs, clinically relevant concentrations of lidocaine, ropivacaine, and bupivacaine inhibited mitochondrial respiration and increased oxidative stress through multiple transcriptionally mediated mechanisms. ${ }^{21}$ In a similar study, when MSCs were exposed to bupivacaine, ropivacaine, or lidocaine, administration of lidocaine was found to have a significant cytotoxic effect. ${ }^{22}$ Moreover, when MSC monolayers were exposed directly to bupivacaine, ropivacaine, and mepivacaine, all three showed time- and concentration-dependent cytotoxic effects. $^{23}$ Based on this in vitro work, it is clear that local anesthetics may have a deleterious effect on the function of MSCs.

These effects may be particularly important for orthopedic surgeries in which local anesthetics are routinely used and where the surgical administration of exogenous MSCs to repair articular defects has been studied. Given the potential role of intra-articular MSCs for tissue healing, ${ }^{24}$ further investigation of the toxicity of local anesthetics on MSCs is warranted. These effects may also apply to patients from whom MSCs are harvested. In a study of lidocaine and adrenaline (commonly used during lipoaspiration) on adipose-derived stem cells, the use of these drugs increased cytotoxicity in a dose- and timedependent manner. ${ }^{25}$ Thus, the judicious use of local anesthetics may be warranted both for patients who have received MSC therapy and for donors from whom MSCs are being harvested.

Limited evidence exists regarding the effects of general anesthetics on MSCs. When bone marrow-derived MSCs 
were treated with sevoflurane or propofol, only sevoflurane exposure resulted in decreased cell viability and changes in cell morphology. ${ }^{26}$ Interestingly, the combination of propofol and sevoflurane at concentrations that were not individually harmful resulted in cytotoxic effects to the MSCs, suggesting that the combined usage of the two anesthetics may have deleterious synergistic effects on MSCs.

The clinical relevance of these limited number of in vitro studies is unclear. Further research is needed to clarify the dose-response relationships as well as their relative and additive toxicities on MSCs. Future studies should also include the use of in vivo models to determine the safety of exposing MSCs to anesthetics.

\section{Future promise of MSCs}

Mesenchymal stromal cells clearly hold promise to fill the unmet perioperative needs of reducing surgical trauma, inflammation, and sepsis while enhancing tissue repair. Collaborative efforts amongst basic scientists, clinical trialists, and bedside clinicians will be needed to further our understanding of these cells and to harness their potential therapeutic benefits to improve perioperative patient outcomes.

\section{Les cellules souches adultes: implications potentielles pour la médecine périopératoire}

En mai 2012, Santé Canada approuvait l'utilisation des cellules stromales mésenchymateuses pour le traitement des réactions graves de greffe contre hôte en pédiatrie. Le Canada devint ainsi le premier pays au monde à approuver une thérapie à partir de cellules souches. ${ }^{\mathrm{A}}$ Cette décision cruciale annonçait l'arrivée d'une nouvelle modalité d'envergure pour le traitement des maladies inflammatoires.

\section{Que sont les cellules stromales mésenchymateuses?}

Depuis leur première description par Friedenstein et coll. il y a plus de 40 ans, ${ }^{1}$ les cellules stromales mésenchymateuses (CSM) ont répondu à plusieurs appellations, notamment celles de cellules souches mésenchymateuses ou « cellules souches adultes ». Friedenstein, pionnier dans l'étude des CSM, a décrit leur adhérence à des boîtes de culture non traitées, leur morphologie fusiforme, ainsi que leur capacité, sous certaines conditions, à se différencier en chondrocytes, en adipocytes et en ostéocytes. Bien que ces critères de base appartiennent toujours à la définition des $\mathrm{CSM}^{2}$ notre compréhension de ces cellules a remarquablement évolué. Il est important de souligner que les CSM, au contraire des cellules souches embryonnaires, ne constituent pas un seul type de cellules capables de se différencier en de multiples types de cellules in vivo; les CSM forment plutôt une population hétérogène de cellules capables d'un vaste éventail d'activités biologiques. Par conséquent, étant donné qu'elles ne présentent pas les caractéristiques de cellules souches prototypiques, on a suggéré l'utilisation du terme « cellule stromale » pour décrire les CSM. ${ }^{3}$

À l'origine, les CSM étaient isolées à partir de moelle osseuse adulte (dans laquelle on pense qu'elles soutiennent l'hématopoï̀se), de tissu adipeux et de gelée de Wharton tirés de cordons ombilicaux. Ces sources sont, encore aujourd'hui, les mieux caractérisées; toutefois, les CSM sont désormais considérées comme des cellules omniprésentes dans littéralement tous les tissus étudiés. ${ }^{4}$ Il y a controverse quant au rôle physiologique de ces cellules dans leurs conditions in vivo d'origine; néanmoins, certains auteurs ont suggéré que les CSM pourraient être des cellules périvasculaires (ou «péricytes ») impliquées dans l'homéostase et la régénération tissulaire. Dans des études précliniques, les CSM périvasculaires semblent être mobilisées dans la circulation lors de conditions inflammatoires telles que les lésions traumatiques des poumons ${ }^{5}$ ou l'hypoxie. ${ }^{6} \mathrm{Ce}$ phénomène a également été observé en clinique chez des patients âgés après une fracture traumatique de la hanche. ${ }^{7}$

\section{Les CSM en tant que traitement 'prêt à l'usage'}

Les cellules stromales mésenchymateuses peuvent être mises en culture et proliférer de façon considérable dans un environnement in vitro, indépendamment de leur source tissulaire. Lorsque leur nombre est suffisamment élevé, les CSM allogéniques peuvent être traitées et administrées à un patient sans vérifier la compatibilité croisée. On a déjà utilisé des cellules allogéniques non appariées dans des applications cliniques; ${ }^{8}$ des cellules xénogéniques ont même été utilisées avec succès dans des modèles précliniques immunocompétents. ${ }^{9}$ On a attribué cette capacité remarquable des CSM à échapper à la détection $\mathrm{du}$ système immunitaire du receveur à l'absence d'un complexe majeur d'histocompatibilité de classe II et de molécules co-stimulantes nécessaires à la reconnaissance des cellules hôtes T CD4+. ${ }^{10}$ Le statut 'immunoprivilégié' des CSM est important d'un point de vue clinique. En effet, c'est l'un des facteurs qui en fait un produit 'prêt à l'usage' qui pourrait être administré dans les circonstances d'urgence. 


\section{Applications périopératoires potentielles des CSM}

Dans les études animales, les effets des CSM administrées de façon exogène semblent largement anti-inflammatoires et de protection des organes; par conséquent, les CSM pourraient potentiellement être bénéfiques contre le stress chirurgical et l'inflammation observés en période périopératoire. Par exemple, dans un modèle préclinique de lésion pulmonaire induite par la ventilation, les CSM administrées par voie intra-trachéale ou intraveineuse ont réduit la production de cytokines pro-inflammatoires et amélioré l'oxygénation artérielle et la compliance pulmonaire. ${ }^{11}$ De la même façon, les CSM administrées dans plusieurs importants modèles animaux d'infarctus du myocarde ont augmenté la fraction d'éjection et réduit la taille de la cicatrice. ${ }^{12}$ Les cellules stromales mésenchymateuses se sont également avérées utiles dans le traitement des lésions rénales aiguës précliniques, où elles ont démontré d'importants effets anti-apoptotiques et cytoprotecteurs. ${ }^{13} \mathrm{Il}$ est intéressant de mentionner que, dans des modèles précliniques de sepsis, les CSM ont non seulement amélioré la survie et la fonction des organes, mais ont également démontré de puissants effets antibactériens. ${ }^{14}$ Étant donné ces effets protecteurs sur les organes ainsi que leur capacité à éliminer les pathogènes, il est clair que les CSM pourraient avoir des bienfaits potentiels pour de nombreux patients en période périopératoire. En période préopératoire, de nombreuses conditions pourraient également potentiellement être améliorées grâce aux CSM (tableau). À ce jour toutefois, la population périopératoire n'a fait l'objet que de quelques études cliniques portant sur les CSM; voilà donc une occasion importante pour la recherche clinique future sur les CSM.

\section{Les voies de transmission thérapeutiques des CSM}

Les mécanismes exacts par lesquels les CSM exercent leurs effets bénéfiques demeurent quelque peu inconnus et

Tableau Exemples de conditions pour lesquelles l'administration de CSM pourrait avoir un potentiel thérapeutique

\begin{tabular}{ll}
\hline Études précliniques & Études cliniques \\
\hline $\begin{array}{l}\text { Lésion pulmonaire induite par } \\
\text { la ventilation }\end{array}$ & $\begin{array}{l}\text { Infarctus du myocarde et insuffisance } \\
\text { cardiaque aigus }\end{array}$ \\
$\begin{array}{l}\text { Hypertension pulmonaire } \\
\text { Lésion pulmonaire aiguë } \\
\text { infectieuse }\end{array}$ & $\begin{array}{l}\text { Réaction de greffe contre hôte } \\
\text { Dysplasie bronchopulmonaire rénale aiguë } \\
\text { Sepsis }\end{array}$ \\
$\begin{array}{l}\text { Traumatismes } \\
\text { Brûlures }\end{array}$ & Sclérose en plaques \\
\hline
\end{tabular}

controversés. Il est toutefois clair que les effets des CSM ne s'appuient pas sur la capacité de ces cellules à se greffer. En effet, la rétention cellulaire totale est extrêmement basse et ce, même lorsque les CSM sont livrées directement dans le tissu (par ex., $<6 \%$ de rétention dix jours après une injection transendocardique). ${ }^{15}$ En revanche, on pense que les CSM migrent par chimiotaxie vers les zones d'inflammation ${ }^{16}$ et ont des effets pléiotropiques via des mécanismes paracrines (figure). Ce cas a été clairement démontré dans un modèle préclinique de sepsis dans lequel des CSM ont été administrées par injection intraveineuse et seuls des niveaux très bas de persistance cellulaire ont pu être établis. ${ }^{17}$ Toutefois, dans cette étude, l'analyse des micro-réseaux d'ARN messager de la rate, des poumons, du cœur et du fois a révélé que 4751 gènes étaient modifiés de façon significative chez les animaux traités avec les CSM par rapport aux animaux traités par placebo. L'analyse des réseaux de ces réponses transcriptionnelles a permis d'identifier des voies de transmission communes servant à améliorer la fonction mitochondriale, freiner la réponse pro-inflammatoire et maintenir l'intégrité endothéliale. Ainsi, à l'inverse des thérapies se fondant sur des médicaments agissant principalement via des mécanismes de 'clé et serrure', les thérapies cellulaires à base de CSM agissent via une multitude de voies de transmission afin de réduire l'inflammation et de maintenir l'homéostase.

Les mécanismes innovants par lesquels les CSM exercent leurs effets thérapeutiques font l'objet d'un intérêt particulier dans les travaux de recherche récents. Un exemple intéressant a été observé dans un modèle murin de lésion pulmonaire aiguë dans lequel des CSM humaines ont été administrées par voie intra-trachéale. Ces CSM exogènes ont formé des canaux de jonction communicante avec les cellules murines hôtes et transféré des mitochondries en formant des microvésicules. ${ }^{18}$ Ces transferts ont été observés par microscope, et on a également noté la présence d'ADN mitochondrial humain persistant dans les poumons murins récipiendaires. D'un point de vue fonctionnel, le transfert mitochondrial a entraîné une augmentation de la formation d'adénosine triphosphate (ATP) et une amélioration de la survie de ces animaux.

Outre les mitochondries, d'autres facteurs libérés par les CSM pourraient aussi être dignes d'intérêt. Des concentrés de facteur peuvent être produits à partir de facteurs libérés par les CSM en récoltant des milieux de culture dans lesquels ces cellules ont été cultivées. Dans des modèles précliniques de lésion pulmonaire aiguë, il a été démontré que ces concentrés de milieux de culture de CSM pouvaient être aussi efficaces que les CSM pour réduire l'inflammation. ${ }^{11,19}$ Des recherches supplémentaires seront nécessaires afin de mieux caractériser les facteurs thérapeutiques libérés par les CSM. 


\section{Innocuité des CSM}

Étant donné les effets potentiellement bénéfiques des CSM, il n'est pas surprenant qu'elles aient été testées pour le traitement de toutes sortes de conditions cliniques (tableau). On a testé les cellules stromales mésenchymateuses dans des études cliniques sur des sujets humains dans diverses conditions, notamment l'accident vasculaire cérébral, l'infarctus du myocarde, l'insuffisance cardiaque, l'insuffisance rénale aiguë et la réaction de greffe contre hôte résistante aux stéroïdes, pour ne citer qu'elles. Tout comme c'est le cas pour tout traitement expérimental, l'innocuité doit demeurer au centre des préoccupations. Afin de tenir compte de ces préoccupations, nous avons récemment réalisé une revue méthodique et une méta-analyse sur l'innocuité des CSM dans les études cliniques. ${ }^{20}$ Dans notre analyse des études publiées, nous n'avons découvert aucune association entre une administration systémique de CSM et des effets secondaires néfastes tels que le dysfonctionnement systémique ou des réactions de perfusion aiguë. Fait intéressant, malgré les effets immunosuppresseurs des CSM, les patients des études n'ont pas montré de taux supérieurs d'infection. Cette constatation s'aligne avec les effets bénéfiques des CSM sur la clairance bactérienne observés dans les modèles précliniques de sepsis. De plus, même si les CSM sont immunoprivilégiées et peuvent se greffer, il n'y a pas eu d'augmentation dans la formation ou la malignité de tumeurs. Cette revue a toutefois été limitée par le faible nombre global d'études et le faible nombre total de patients étudiés, par les périodes relativement courtes de suivi ainsi que par la variété des schémas de dosage des CSM. Des études cliniques bien réalisées et rigoureuses supplémentaires seront nécessaires afin de clarifier plus avant le profil d'innocuité des CSM. Dans les études futures, il faudra également examiner attentivement la relation de dose-réponse des CSM dans diverses conditions.

\section{Implications potentielles des agents anesthésiques sur les CSM}

Une considération importante pour les anesthésiologistes quant à l'utilisation thérapeutique des CSM réside dans l'effet potentiel des anesthésiques locaux et généraux sur ces cellules. Ceci aura de l'importance pour les patients futurs qui reçoivent des CSM en période préopératoire et peropératoire.

L'utilisation périopératoire d'anesthésiques locaux pourrait poser problème car ces médicaments sont cytotoxiques pour plusieurs types de cellules, et il semble que les CSM pourraient également y être susceptibles.
Dans une étude portant sur des CSM murines, des concentrations pertinentes d'un point de vue clinique de lidocaïne, de ropivacaïne et de bupivacaïne ont inhibé la respiration mitochondriale et augmenté le stress oxydatif par le biais de nombreux mécanismes médiés de façon transcriptionnelle. ${ }^{21}$ Dans une étude semblable, lorsque des CSM ont été exposées à la bupivacaïne, à la ropivacaïne ou à la lidocaïne, on a observé que l'administration de lidocaïne avait un effet cytotoxique significatif. ${ }^{22}$ En outre, lorsque des monocouches de CSM ont été exposées directement à de la bupivacaïne, de la ropivacaïne ou de la mépivacaïne, ces trois agents ont tous démontré des effets cytotoxiques dépendants de la durée et de la concentration. ${ }^{23}$ Sur la base de ces travaux in vitro, il est clair que les anesthésiques locaux pourraient avoir un effet délétère sur la fonction des CSM.

Ces effets pourraient être particulièrement importants dans les cas de chirurgies orthopédiques, au cours desquelles les anesthésiques locaux sont fréquemment utilisés et où l'administration chirurgicale de CSM exogènes pour réparer les défauts articulaires a été étudiée. Étant donné le rôle potentiel des CSM intra-articulaires pour la guérison tissulaire, des recherches supplémentaires portant sur la toxicité des anesthésiques locaux sur les CSM sont nécessaires. Ces effets pourraient également s'appliquer aux patients desquels les CSM sont prélevées. Dans une étude portant sur les effets de la lidocaïne et de l'adrénaline (deux agents fréquemment utilisés pendant les liposuccions) sur les cellules souches dérivées de tissu adipeux, l'utilisation de ces médicaments a augmenté la cytotoxicité de façon dépendante à la dose et à la durée. ${ }^{25}$ Pour toutes ces raisons, l'utilisation judicieuse d'anesthésiques locaux est de mise, aussi bien chez les patients ayant reçu un traitement à base de CSM que chez les donneurs desquels des CSM ont été prélevées.

Des données probantes limitées existent concernant les effets des anesthésiques généraux sur les CSM. Lorsqu'on a traité des CSM dérivées de la moelle osseuse au sévoflurane ou au propofol, seule l'exposition au sévoflurane a entraîné une réduction de la viabilité des cellules et des modifications au niveau de leur morphologie. ${ }^{26}$ Fait intéressant, la combinaison de propofol et de sévoflurane à des concentrations qui n'étaient pas nocives individuellement a eu des effets cytotoxiques sur les CSM, ce qui laisse penser que l'utilisation combinée de ces deux agents anesthésiques pourrait avoir des effets synergistes délétères sur les CSM.

La pertinence clinique de ce nombre limité d'études in vitro n'est pas claire. Des recherches supplémentaires sont nécessaires pour clarifier les relations de dose-réponse ainsi que leurs toxicités relatives et additives sur les CSM. Les études futures devront également s'intéresser à l'utilisation de modèles in vivo pour déterminer s'il est sécuritaire d'exposer les CSM aux agents anesthésiques. 


\section{Les promesses futures des CSM}

Les cellules stromales mésenchymateuses sont indubitablement prometteuses pour répondre aux besoins périopératoires non comblés que sont la réduction des traumatismes, de l'inflammation et du sepsis tout en améliorant la régénération des tissus. La collaboration sera nécessaire entre scientifiques fondamentaux, chercheurs cliniques et cliniciens au chevet afin de faire avancer notre compréhension de ces cellules et saisir leurs bienfaits thérapeutiques potentiels, de façon à améliorer les pronostics périopératoires des patients.

Conflicts of interest None declared.

Conflit d'intérêt Aucun.

\section{References}

1. Friedenstein AJ, Petrakova KV, Kurolesova AI, Frolova GP. Heterotopic of bone marrow. Analysis of precursor cells for osteogenic and hematopoietic tissues. Transplantation 1968; 6: 230-47.

2. Dominici M, Le Blanc K, Mueller I, et al. Minimal criteria for defining multipotent mesenchymal stromal cells. The International Society for Cellular Therapy position statement. Cytotherapy 2006; 8: 315-7.

3. Horwitz EM, Le Blanc K, Dominici M, et al. Clarification of the nomenclature for MSC: The International Society for Cellular Therapy position statement. Cytotherapy 2005; 7: 393-5.

4. da Silva Meirelles L, Chagastelles PC, Nardi NB. Mesenchymal stem cells reside in virtually all post-natal organs and tissues. J Cell Sci 2006; 119(pt 11): 2204-13.

5. Hannoush EJ, Sifri ZC, Elhassan IO, et al. Impact of enhanced mobilization of bone marrow derived cells to site of injury. $\mathrm{J}$ Trauma 2011; 71: 283-9.

6. Rochefort GY, Delorme B, Lopez A, et al. Multipotential mesenchymal stem cells are mobilized into peripheral blood by hypoxia. Stem Cells 2006; 24: 2202-8.

7. Alm JJ, Koivu HM, Heino TJ, Hentunen TA, Laitinen S, Aro HT. Circulating plastic adherent mesenchymal stem cells in aged hip fracture patients. J Orthop Res 2010; 28: 1634-42.

8. Le Blanc K, Frassoni F, Ball L, et al. Mesenchymal stem cells for treatment of steroid-resistant, severe, acute graft-versus-host disease: a phase II study. Lancet 2008; 371: 1579-86.

9. Gonzalez-Rey E, Anderson P, Gonzalez MA, Rico L, Buscher D, Delgado $M$. Human adult stem cells derived from adipose tissue protect against experimental colitis and sepsis. Gut 2009; 58: 929-39.

10. Pittenger MF, Mackay AM, Beck SC, et al. Multilineage potential of adult human mesenchymal stem cells. Science 1999; 284: 143-7.
11. Curley GF, Ansari B, Hayes M, et al. Effects of intratracheal mesenchymal stromal cell therapy during recovery and resolution after ventilator-induced lung injury. Anesthesiology 2013; 118 : 924-32.

12. Williams AR, Hare JM. Mesenchymal stem cells: biology, pathophysiology, translational findings, and therapeutic implications for cardiac disease. Circ Res 2011; 109: 923-40.

13. Togel F, Weiss $K$, Yang $Y, H u ~ Z$, Zhang P, Westenfelder $C$. Vasculotropic, paracrine actions of infused mesenchymal stem cells are important to the recovery from acute kidney injury. Am J Physiol Renal Physiol 2007; 292: F1626-35.

14. Mei SH, Haitsma JJ, Dos Santos CC, et al. Mesenchymal stem cells reduce inflammation while enhancing bacterial clearance and improving survival in sepsis. Am J Respir Crit Care Med 2010; 182: 1047-57.

15. Gyongyosi M, Blanco J, Marian $T$, et al. Serial noninvasive in vivo positron emission tomographic tracking of percutaneously intramyocardially injected autologous porcine mesenchymal stem cells modified for transgene reporter gene expression. Circ Cardiovasc Imaging 2008; 1: 94-103.

16. Chapel A, Bertho JM, Bensidhoum M, et al. Mesenchymal stem cells home to injured tissues when co-infused with hematopoietic cells to treat a radiation-induced multi-organ failure syndrome. J Gene Med 2003; 5: 1028-38.

17. dos Santos CC, Murthy S, Hu P, et al. Network analysis of transcriptional responses induced by mesenchymal stem cell treatment of experimental sepsis. Am J Pathol 2012; 181: 168192.

18. Islam MN, Das SR, Emin MT, et al. Mitochondrial transfer from bone-marrow-derived stromal cells to pulmonary alveoli protects against acute lung injury. Nat Med 2012; 18: 759-65.

19. Ionescu L, Byrne RN, van Haaften T, et al. Stem cell conditioned medium improves acute lung injury in mice: in vivo evidence for stem cell paracrine action. Am J Physiol Lung Cell Mol Physiol 2012; 303: L967-77.

20. Lalu MM, McIntyre L, Pugliese $C$, et al. Safety of cell therapy with mesenchymal stromal cells (SafeCell): a systematic review and meta-analysis of clinical trials. PLoS One 2012; 7: e47559.

21. Lucchinetti E, Awad AE, Rahman M, et al. Antiproliferative effects of local anesthetics on mesenchymal stem cells: potential implications for tumor spreading and wound healing. Anesthesiology 2012; 116: 841-56.

22. Rahnama $R$, Wang $M$, Dang AC, Kim HT, Kuo AC. Cytotoxicity of local anesthetics on human mesenchymal stem cells. J Bone Joint Surg Am 2013; 95: 132-7.

23. Breu A, Eckl S, Zink W, Kujat R, Angele P. Cytotoxicity of local anesthetics on human mesenchymal stem cells in vitro. Arthroscopy 2013; 29: 1676-84.

24. Orozco L, Munar A, Soler $R$, et al. Treatment of knee osteoarthritis with autologous mesenchymal stem cells: a pilot study. Transplantation 2013; 95: 1535-41.

25. Girard AC, Atlan $M$, Bencharif $K$, et al. New insights into lidocaine and adrenaline effects on human adipose stem cells. Aesthetic Plast Surg 2013; 37: 144-52.

26. Zhou X, Li YQ, He W, et al. Effects of sevoflurane and propofol on cultured bone-marrow mesenchymal stem cells of rats. Int $\mathrm{J}$ Clin Pharmacol Ther 2013; 51: 332-7. 